\title{
Tagungsbericht
}

Maike Neufend und Maxi Kindling

\section{Von Open Access zu Open Science und wieder zurück?}

\section{From Open Access to Open Science and back again?}

\section{Bericht über den Stand der Open-Access-Transformation im Rückblick auf die Open-Access- Tage 2020}

\author{
A Report on the Status of Open-Access-Transformation in Review of the Open Access Days 2020
}

http://doi.org/10.1515/abitech-2021-0007

\section{1 „At home but open“}

Der offene und damit auch kostenfreie Zugang zu wissenschaftlichen Ergebnissen ist das wichtigste Prinzip von Open Access. Die diesjährigen Open-Access-Tage, die vom 15. bis 17. September 2020 stattgefunden haben, lieferten mit der Leitlinie „At home but open“ eine Version der größten deutschsprachigen Konferenz der Open-Accessund Open-Science-Community aus Deutschland, Österreich und der Schweiz, die diesem Prinzip treu geblieben ist. Im digitalen Raum diskutierten teilweise bis zu 330 Personen gleichzeitig ohne Zugangsbarrieren über aktuelle Themen und Entwicklungen der offenen Wissenschaft. Unter dem Motto „Wege, Akteur`innen, Effekte“ legte die Tagung einen Schwerpunkt auf die Rolle von Open Access für Open Science, auf Wissenschaftlerinnen und Wissenschaftler als Open-Access-Akteurinnen und-Akteure sowie auf die Herausforderungen, denen sich unterschiedliche Hochschultypen und Bildungseinrichtungen in der Transformation gegenübersehen. Formate wie Keynotes, Sessions, Workshops und der Tool-Marktplatz bewährten sich auch im digitalen Raum. Ohne die vielen Menschen, die sich auf Seiten der Organisatorinnen und Organisatoren von der Universitätsbibliothek Bielefeld und der Hochschulbibliothek der Fachhochschule Bielefeld um den reibungslosen Ablauf kümmerten, wäre auch die Online-Ausgabe der Open-Access-Tage nicht mit diesem Erfolg möglich gewesen. An den Bedarf einer Online-Tagung angepasst, begleitete der Hochschulsport der Universität Bielefeld die Tagung über die drei Tage hinweg mit einem Pausenexpress in Form kleiner Bewegungsein- heiten. Abgerundet wurde das Rahmenprogramm durch die virtuelle Happy Hour „Bring Your Own Bottle“ und die Lesung von Elio Pellin aus dem Rätselroman Der Himmel als Abgrund über euch.

\section{Von Open Access zu Open Science: Strategien, Infrastruk- turen und Herausforderungen}

Die nationalen und europäischen Bemühungen um eine umfassende Open-Access-Transformation im Bereich Publikationen sind bereits weit fortgeschritten. Für viele Strategien und Policies ist 2020 ein wichtiges Jahr, in dem unter anderem die EU-Mitglieder-Quoten von $100 \%$ Open Access bei Zeitschriftenartikeln umgesetzt sein sollen. „Die Transformationsinitiative OA2020 trägt das Jahr sogar im Namen“, schreiben Andrea Hacker und Marco Tullney in ihrem Beitrag „Stell Dir vor, es ist 2020 und Open Access läuft immer noch nicht" ". Auch wenn einige Förderer Open Access zur Pflicht gemacht haben und Mittel zur Verfügung gestellt werden, wurden viele Ziele im Jahr 2020 nicht erreicht. Hacker und Tullney sprachen sogar von einer „Misere von überhöhten APCs, Publisher-led-Entwicklungen, fehlender nachhaltiger und ernstzunehmender infrastruktureller Publikationsalternativen und dem noch immer erdrückenden Fokus auf

1 Hacker, Andrea und Marco Tullney. „Stell Dir vor, es ist 2020 und Open Access läuft immer noch nicht." Gehalten auf: Open-AccessTage 2020 (OAT2020), 15. September 2020, Bielefeld. doi:10.5281/ zenodo.4028988. 
Zeitschriftenartikel“.2 Insbesondere die Reputationsökonomie sei zum großen Teil nicht in Richtung Open Access weiterentwickelt worden, so dass weiterhin der Druck auf den Forschenden lastet, in Closed-Access-, aber zugleich renommierten Publikationen zu veröffentlichen. Die europäische Initiative Plan S, die auch Forschende individuell anspricht und eine Strategie zur Veränderung der Reputationsökonomie anregt, spiele, so Tullney, in der deutschen Wissenschaftslandschaft für die Institutionen keine große Rolle. ${ }^{3}$ Ein Blick auf die Unterzeichnenden der DORA-Erklärung (San Francisco Declaration on Research Assessment) zeigt zudem, dass sie in Deutschland keineswegs breit unterstützt wird. ${ }^{4}$ Wie können wir also aus dieser Misere entkommen?

Ein Weg, der sich auf nationaler und europäischer Ebene abzeichnet, ist die Öffnung der Debatte hin zu Open Research oder Open Science und damit zu einer breiteren Diskussion über die Bedeutung von Offenheit im Forschungsprozess. Die Keynote-Sprecherin Sabina Leonelli (University of Exeter) zeigte in ihrem Vortrag „Opening the Research Process: From Publications to Data, and Back Again ${ }^{\star 5}$ anschaulich, wie Open Science im besten Fall zu einer „platform for critical, informed and open debate“ werden kann. ${ }^{6}$ Der Bericht der „Open Science Policy Platform“ im Horizon 2020 Programm der Europäischen Union weist unter anderem auf die Notwendigkeit hin, dass Anreize und Belohnungen für das Engagement im Bereich Open Science geschaffen werden müssen. ${ }^{7}$ Im Detail spricht dieser Bericht von einem ,wholesale change to the reward and tenure system". ${ }^{8}$ Der Wechsel hin zu Open Science bringt jedoch mehr noch als Open Access für unterschiedliche Disziplinen sehr unterschiedliche Herausforderungen. Während Susanne Blumesberger von der Universität Wien dazu aufrief, Open Science in kleinen Schritten den Forschenden durch Aufklärung und Beratung nahe zu bringen, ${ }^{9}$ betonte Sabina Leonelli, dass die disziplinären Traditionen nicht die Hinderungsgründe

2 Hacker/Tullney 2020.

3 Hacker/Tullney 2020: Folie 9.

4 Hacker/Tullney 2020: Folie 8.

5 Leonelli, Sabina. „Opening the Research Process: From Publications to Data, and Back Again.“ Gehalten auf: Open-Access-Tage 2020 (OAT2020), 17. September 2020, Bielefeld. doi:10.5281/zenodo. 4045738.

6 Leonelli 2020: Folie 27.

7 Leonelli 2020: Folie 12.

8 European Commission. Directorate General for Research and Innovation. Progress on open science: towards a shared research knowledge system: final report of the open science policy platform. LU: Publications Office, 2020. doi:10.2777/00139, 23.

9 Blumesberger, Susanne. „Auf dem Weg zu Open Science in kleinen Schritten.“ 7. Oktober 2020. doi:10.5281/zenodo.4070751. darstellen, denn bereits die Methoden und Gewohnheiten sind in jedem Forschungsbereich sehr divers. ${ }^{10}$ Diese diversen Perspektiven gilt es zu schätzen und damit einen Dialog darüber anzuregen, was als Wissenschaft zählt und wie Ergebnisse anerkannt werden, betont Leonelli. ${ }^{11}$ Open Science ist als Chance zu sehen, die aber nur durch Change erreicht werden kann.

Durch datengetriebene Infrastruktur-Initiativen wie die European Open Science Cloud (EOSC) und die Nationale Forschungsdateninfrastruktur (NFDI) sollen Infrastrukturen und Dienstleistungen integriert werden. Auch in diesen Initiativen auf europäischer und nationaler Ebene besitzt laut OpenAIRE der offene Zugang zu Forschungsergebnissen Priorität. ${ }^{12}$ Ziel der NFDI ist es, in den nächsten 15-20 Jahren ein neues Level von Dateninfrastrukturen, neue Berufsprofile, wie beispielsweise Datenbibliothekarinnen und Datenbibliothekare, und eine nachhaltige Förderrichtlinie aufzubauen. ${ }^{13}$ OpenAIRE nimmt zwischen der EOSC und der NFDI eine komplementäre Rolle ein, unter anderem auch um Training und Unterstützung im Bereich Open Science anzubieten und damit einen kulturellen Wandel in Richtung Open Science zu unterstützen. ${ }^{14}$ Diese Entwicklung von Open Access hin zu Open Science zeigte sich im Rahmen der Tagung auch bei zwei weiteren Beiträgen: In der Schweiz wird momen$\tan$ an einer Open-Science-Strategie gearbeitet ${ }^{15}$ und die Berlin-Brandenburgische Akademie der Wissenschaften (BBAW) hat 2019 ein „Leitbild Open Science“ verabschiedet. $^{16}$

Wie divers die Umsetzung von Open Access und Open Science in Deutschland ist, zeigte der Workshop zu Open Access in den einzelnen Bundesländern. Dabei wird deutlich, dass auch dort die Vielfalt der Publikationskulturen einzelner Fachbereiche und Einrichtungen als Herausforderung wahrgenommen wird. Unterstützend wirkt die

10 Leonelli 2020: Folie 18.

11 Leonelli 2020: Folie 27.

12 Czerniak, Andreas, Lena Dreher, Emilie Hermans u.a. „Wie befördern NFDI und EOSC einen kulturellen Wandel für eine offene Wissenschaft?“ Gehalten auf: Open-Access-Tage 2020 (OAT2020), 15. September 2020, Bielefeld, Germany. doi:10.5281/zenodo.4062376, Folie 7.

13 Czerniak u.a. 2020: Folie 10.

14 Czerniak u. a. 2020.

15 Felder, Fabian und Wilfried Lochbühler. „Update Schweiz: Nationale Open Access Strategie und Verhandlungen mit Grossverlagen.“ Gehalten auf: Open-Access-Tage 2020 (OAT2020), 15. September 2020, Bielefeld, Germany. doi:10.5281/zenodo.4063446.

16 Seidig, Marianne und Markus Schnöpf. „Open Science an der Berlin-Brandenburgischen Akademie der Wissenschaften (BBAW)." Gehalten auf: Open-Access-Tage 2020 (OAT2020), 16. September 2020, Bielefeld, Germany. doi:10.5281/zenodo.4045655. 
Verankerung von praxisorientierten Leitlinien in Open-Access-Strategien, um Wissenschaftlerinnen und Wissenschaftler für eine stärkere und aktivere Wahrnehmung von Open-Access-Publikationsoptionen zu ermutigen. Das Projekt Bundesländer-Atlas im Rahmen des BMBF-geförderten Projekts open-access.network beleuchtet die Ansätze, die auf Landesebene besonders effektiv wirken. Damit können wissenschaftspolitischen Akteurinnen und Akteuren der bundes- und landespolitischen Ebene sowie den Open-Access-Professionals Informationen über Entwicklungen und gute Praktiken in anderen Bundesländern bereitgestellt werden. ${ }^{17}$ Ein wichtiges Projekt auf Landesebene ist openaccess.nrw, das Vorprojekt zur Digitalen Hochschule NRW (DH.NRW) ${ }^{18}$ Anhand von qualitativen und quantitativen Studien wurden die Service-Bedarfe an Hochschulen in Nordrhein-Westfalen erhoben. In ihrem Workshop zu „Umsetzungsoptionen für Open-Access-Services“ erarbeiteten die Teilnehmenden Best- und Worst-Case-Szenarien. Die Ergebnisse des Workshops in Form einer Tabelle geben einen guten Eindruck von den möglichen Services und Maßnahmen, die insbesondere für die Entwicklung von Landesstrategien sinnvoll sind. ${ }^{19}$

\section{Wissenschaftlerinnen und Wissenschaftler als Open- Access-Akteurinnen und-Akteure: die Herausforderungen des Scholar-led Publishing}

Die großen Initiativen auf nationaler und europäischer Ebene zeigen die Bedeutung der Entwicklung hin zu Open Access und Open Science im Wissenschaftssystem. Als ein wichtiger Aspekt der Open-Access-Transformation ist das nicht kommerzielle Scholar-led Publishing jedoch kaum Teil der größeren Debatten. Dauerhaft unterfinanziert, ist dieses Segment deshalb häufig gezwungen, Geschäftsmodelle zu adaptieren, die letztlich kommerziellen Logiken entsprechen. ${ }^{20}$ „It is possible to run journal publishing on

17 Eppelin, Anita, Ben Kaden, Maxi Kindling u. a. „Open Access in den deutschen Bundesländern - Workshop von und für die Landesinitiativen Bericht.“ Zenodo (2020). doi:10.5281/zenodo.4058071.

18 DH.NRW: Startseite. https://www.dh.nrw/ (27.11.2020).

19 Schulz, Katharina. „Ergebnisse des Workshops bei den Open-Access-Tagen 2020 - openaccess.nrw. 6. November 2020. https://open access.nrw/?p=253 (27.11.2020).

20 Bergmann, Max, Kathrin Ganz, Maike Neufend u.a. „Open Access: Es ist Zeit für VerlegerInnen aus der Wissenschaft." Digital Soci- a not-for-profit basis and in hands of the academic sector, naturally open to readers and free of APCs“, ${ }^{21}$ betont die Keynote-Sprecherin Arianna Becerril García. Als Mitglied von AmeliCA $^{22}$ berichtete sie aus lateinamerikanischer Perspektive über den nicht-kommerziellen Zugang zu Wissenschaft durch kooperative Ansätze, „where everyone gets benefit from everyone's investment“. Auf beeindruckende Art und Weise hat Lateinamerika eine nicht-kommerzielle Publikationsinfrastruktur aufgebaut und etabliert, in der das Publikationswesen der Wissenschaft gehört, und nicht den großen Verlagen. Diese Infrastruktur ist laut Arianna Becerril García möglich, weil Wissen und Wissenschaft als commons behandelt werden, das heißt, 96,45\% der wissenschaftlichen Publikationen in Lateinamerika sind nicht-kommerziell und werden von Fachgemeinschaften, Universitäten, Regierungen und Krankenhäusern finanziert. Ebenso wie Universitäten und Forschungseinrichtungen öffentlich finanziert werden, wird auch die Kommunikation von Wissen öffentlich finanziert. ${ }^{23}$

Die wissenschaftliche Publikationslandschaft in Deutschland unterscheidet sich sehr stark von diesem kooperativen Modell. Das DFG-finanzierte Projekt „Innovative Open Access in small sciences“ (InnOAccess) ${ }^{24}$ hat eine Umfrage zur Lage von verlagsunabhängigen, gebührenfreien Zeitschriften durchgeführt, durch die eine diverse Publikationskultur in Deutschland bestätigt werden konnte. Es scheint nicht die eine Lösung für alle Einrichtungen zu geben, weshalb ein modulares, bedarfsorientiertes Finanzierungskonzept vorgeschlagen wird. Ähnlich wie die Publikationsinfrastrukturen in Lateinamerika, ${ }^{25}$ die nicht-kommerzielles wissenschaftliches Publizieren unterstützen, argumentiert auch das Projekt InnOAccess für nachhaltige und kooperative technische Lösungen.

ety Blog. HIIG. 20. November 2019. https://www.hiig.de/open-accesses-ist-zeit-fuer-verleger-aus-der-forschung/ (10.11.2020).

21 Becerril García, Arianna. „Non-commercial Open Access to science, the closest approach toward sustainable and participatory scholarly communications.“ Gehalten auf: Open-Access-Tage 2020 (OAT2020), 16. September 2020, Bielefeld. doi:10.5281/zenodo. 4045693.

22 AmeliCA ,is a communication infrastructure for scholarly publishing and open science. Sustained cooperatively, this initiative focuses on a non-profit publishing model to preserve the scholarly and open nature of scientific communication": http://amelica.org/index. php/en/about/\#quienes-somos (10.11.2020).

23 Becerril García 2020: Folie 12.

24 Siehe Projektbeschreibung: „Innovative Open Access in small sciences (InnOAccess).“ 2020: https://www.hiig.de/en/project/ innoaccess/ (10.11.2020).

25 Siehe beispielsweise: https://www.redalyc.org/ (10.11.2020). 
Eine innovative Verbindung von Publikationen und Forschungsdaten stellte Peter Baumeister vom Deutschen Archäologischen Institut (DAI) vor. Für den Relaunch des Archäologischen Anzeigers wurde ein neues Publikationsformat entwickelt, bei dem Artikel mit Forschungsdaten in Form digitaler Supplemente verschränkt werden. Der DAI-Journal-Viewer basiert auf einer Open Source Software ${ }^{26}$ und kann strukturierte Datenkonvolute aus Quellsystemen der iDAI.world in die angezeigten Beiträge einbinden. ${ }^{27}$ Damit wird Forschenden nicht nur die Möglichkeit gegeben, ihre Ergebnisse im Open Access zugänglich zu machen, sondern auch ihre Artikel mit Forschungsdaten anzureichern, womit die Sichtbarkeit der eigenen Publikations- und Forschungsleistung erhöht wird. ${ }^{28}$ Dass Datenformate der Open-Access-Publikationen offener gestaltet werden müssen, also jenseits der PDF-Publikation, argumentierten auch Christof Schöch und Maria Hinzmann von der Universität Trier. In Zukunft seien Forschungsergebnisse „nicht mehr nur in natürlichsprachiger Prosa formuliert“, sondern Code und Datensätze verbinden sich mit dem Text, so dass dieser mit reichhaltigen Metadaten und strukturierten bibliographischen Daten versehen wird. ${ }^{29}$

Auch für diese technischen Lösungen spielt die Finanzierung eine bedeutende Rolle. Dabei könnten die Bibliotheken durch Konsortienmodelle eine maßgebliche Funktion übernehmen, um Finanzierungsnetzwerke für kooperatives und gebührenfreies Open Access zu gestalten. ${ }^{30}$ Der Open-Access-Dienstleister Knowledge Un-

26 Siehe beispielsweise: O’Donoghue, Geoff P, Rafal M Pielak, Alexander A Smoligovets u.a. „Direct single molecule measurement of TCR triggering by agonist pMHC in living primary T cells.“ eLife 2 (3. Juli 2013). doi:10.7554/eLife.00778.

27 Siehe beispielsweise: Ardeleanu, Stefan, Moheddine Chaouali, Werner Eck u.a. „Die frühkaiserzeitlichen Grabsteine aus Simitthus (Chimtou). Stilistisch-epigraphische Analyse und urbaner Kontext.“ Archäologischer Anzeiger (18. November 2019): § 1-46-§ 1-46. doi:10.34780/639r-3561.

28 Baumeister, Peter. „Ein neues Zeitschriftenmodell mit integrierten Forschungsdatensupplementen in den Altertumswissenschaften: Der neue Archäologische Anzeiger.“ Gehalten auf: Open-Access-Tage 2020 (OAT2020), 15. September 2020, Bielefeld. https:// www.conftool.org/openaccesstage2020/index.php?page=browseSes sions\&presentations=show\&search=Baumeister (10.11.2020).

29 Schöch, Christof und Maria Hinzmann. „Offene Publikationsformate für Open Science.“ Gehalten auf: Open-Access-Tage 2020 (OAT2020), 15. September2020, Bielefeld. https://www.conftool.org/ openaccesstage2020/index.php?page=browseSessions\&presentatio ns=show\&search=Sch\%C3\%B6ch (10.11.2020).

30 Wrzesinski, Marcel und Kathrin Ganz. „Gebührenfreie Open-Access-Modelle: Update und Roadmap zu einer gemeinschaftlichen Finanzierung.“ Workshop organisiert auf: Open-Access-Tage 2020 (OAT2020), 15. September 2020, Bielefeld. https://www.conftool. latched ${ }^{31}$ betont zudem, dass institutionell finanziertes Open Access für Zeitschriften und Bücher seit 2012 immer mehr Unterstützung erfährt. Diese Entwicklung gilt es durch kollaborative Modelle der Kostenteilung und eine einheitliche Verbreitung unter anderem durch gute Metadaten weiter zu fördern. ${ }^{32}$ Kathrin Ganz (Freie Universität Berlin) konnte durch eine qualitative Studie im Rahmen des Projekts „Open Gender Platform“333 zur Bedeutung von Open Access in den Sozial- und Geisteswissenschaften herausstellen, dass Scholar-led Open-Access-Projekte über die Anschubfinanzierungen hinaus gefördert werden müssen, um diese konkurrenzfähig zu machen und weiter zu professionalisieren. Erst dadurch kann eine Arbeitsteilung im wissenschaftlichen Publizieren an Universitäten und Forschungseinrichtungen gestärkt werden. ${ }^{34}$ Für die Medienwissenschaft betonten Dietmar Kammerer und Sarah-Mai Dang, dass die Multizuständigkeit von Wissenschaftlerinnen und Wissenschaftlern nicht nur als Überforderung gilt, sondern auch dazu führt, dass es ein Informationsdefizit auf Seiten der Redaktionen geben kann. ${ }^{35}$ Es lässt sich zusammenfassen, dass die Open-Access-Tage 2020 erneut gezeigt haben, dass durch Wissenschaftlerinnen und Wissenschaftler selbst verantwortete Publikationsprozesse eine wichtige Säule der Open-Access-Gemeinschaft darstellen. In manchen Regionen außerhalb des europäischen Kontextes ist diese Säule bereits tragend für die Wissenschaftskommunikation. In Deutschland gilt es, diese weiter zu stärken, auch durch Finanzierungsmodelle, die außerhalb kommerzieller Open-Access-Ökonomien wirken.

org/openaccesstage2020/index.php?page=browseSessions\&form session $=83$ \&presentations=show (10.11.2020) und Jobmann, Alexandra. „Workshop (Bibliotheks-)Konsortien für gebührenfreies Open Access.“ 15. Juni 2020. https://oa2020-de.org/blog/2020/06/15/ workshop_konsortien_gebuehrenfreiesOA/ (15.11.2020).

31 Siehe die Webseite: https://knowledgeunlatched.org/ (10.11.2020). 32 Hess, Philipp und Catherine Anderson. „Institutionelles Open Access - Was funktioniert und was nicht funktioniert.“ Gehalten auf: Open-Access-Tage 2020 (OAT2020), 16. September 2020, Bielefeld. doi:10.5281/zenodo.4086027.

33 Siehe die Webseite: https://opengenderjournal.de/ (10.11.2020). 34 Ganz, Kathrin. „Alles Open oder was? Erwartungen, Missverständnisse und Prioritäten von Autor`innen in den Sozial- und Geisteswissenschaften.“ 18. September 2020. doi:10.5281/zenodo. 4037226.

35 Kammerer, Dietmar und Sarah-Mai Dang. „Open Access in der Medienwissenschaft - Akteur`innen, Chancen, Herausforderungen." Gehalten auf: Open-Access-Tage 2020 (OAT2020), 16. September 2020, Bielefeld. doi:10.5281/zenodo.4088662. 


\section{Die Transformation und deren Auswirkungen: Open Access für diverse Hochschultypen, Bildungseinrichtungen und Publikationskulturen}

Den Einstieg in die Open-Access-Tage gestaltete Pierre Mounier mit seiner Keynote zu dem wichtigen Thema der Bibliodiversität. Unter dem Titel „Supporting bibliodiversity in open science: a European challenge“, sprach der Direktor von OpenEdition ${ }^{36}$ über die Bedeutung von Bibliodiversität und die Rolle der Vielfalt in der Publikationslandschaft. Bisher ist dieser Begriff, der zunächst von chilenischen Verlegern Ende der 1990er Jahre genutzt wurde, kaum in den nationalen und europäischen Strategien und Policies zu Open Science vorhanden. ${ }^{37}$ Im Jahr 2018 formulierte ein Kollektiv französischer Forscherinnen und Forscher sowie Fachleute aus dem Verlagswesen den ,Jussieu-Appell für offene Wissenschaft und Bibliodiversität“, der bis heute von 71 Institutionen und 25 wissenschaftlichen Verlagen und Gesellschaften unterzeichnet wurde. ${ }^{38}$ In Deutschland haben nur die Max Weber Stiftung und LIBREAS e.V. (Verein zur Förderung der bibliotheks- und informationswissenschaftlichen Kommunikation) den Aufruf unterschrieben. Am 15. April 2020 veröffentlichte die Confederation of Open Access Repositories (COAR) ${ }^{39}$ einen weiteren „call for action“, in dem es heißt: „the current crisis has exposed the deficiencies in a system that is increasingly homogenous and prioritizes profits over the public good“. ${ }^{40}$ Mounier betonte, dass unter anderem lokale und nationale Werte priorisiert werden, anstatt der Vielfalt im wissenschaftlichen Kommunikationssystem gerecht zu werden. ${ }^{41}$ Dabei zeichnet sich in der Open-Access- und Open-Science-Transformation bereits ab, dass unterschiedliche Hochschultypen und Bildungseinrichtungen spezifische Herausforderungen $\mathrm{zu}$ stemmen

36 OpenEdition ist eine Plattform, die sich elektronischen Ressourcen in den Geistes- und Sozialwissenschaften widmet. Siehe: https:// www.openedition.org/?lang=en (10.11.2020).

37 Mounier, Pierre. „Supporting bibliodiversity in open science: a European challenge.“ Gehalten auf: Open-Access-Tage 2020 (OAT2020), 15. September 2020, Bielefeld. doi:10.5281/zenodo.4045687.

38 Jussieu-Appell für offene Wissenschaft und Bibliodiversität. 2018. https://jussieucall.org/jussieu-appell/ (18.11.2020).

39 Siehe die Webseite: https://www.coar-repositories.org/ (10.11. 2020).

40 Shearer, Kathleen, Leslie Chan, Iryna Kuchma u. a. „Fostering Bibliodiversity in Scholarly Communications: A Call for Action." Zenodo (2020). doi:10.5281/zenodo.3752923.

41 Mounier 2020: Folie 22. haben. Insbesondere Bibliotheken können einen wichtigen Beitrag leisten, Standardmodelle und Kriterien zu entwickeln, um alternative Finanzierungsmodelle abseits von „pay for access“ oder „pay to publish“ zu schaffen. Ulrike Kändler (TIB Hannover) und Elena Di Rosa (TU Berlin) berichten in ihrer Session über freiwillige und gemeinschaftliche Open-Access-Finanzierungsmodelle und deren Bedeutung in wissenschaftlichen Bibliotheken. Faktoren und Entscheidungsprozesse wurden durch eine Umfrage evaluiert, um unter anderem die Rolle APC-freier Modelle im strategischen Handeln der Einrichtungen zu beleuchten. ${ }^{42}$ Festzuhalten ist, dass erst indem Bibliotheken in diverse Inhalte und Dienstleistungen investieren, offene und diverse Infrastrukturen unterstützt werden können. ${ }^{43}$

Bibliotheken sind demnach wichtige Akteure in der Transformation, doch die Herausforderungen sind ebenso divers wie die Bibliothekstypen. In einer Umfrage, die auf Anregung der Arbeitsgemeinschaft Regionalbibliotheken entstanden ist und von Corinna Roeder und Wolf Christoph Seifert (beide Landesbibliothek Oldenburg) vorgestellt wurde, werden Probleme identifiziert, die mit der Herausforderung der Transformation verbunden sind. Dazu gehören unter anderem die eingeschränkten Ressourcen bei der IT-Infrastruktur und auch dem Personal, rechtliche Unsicherheiten sowie Probleme bei der Qualitätssicherung im Bereich Publikationen. ${ }^{44}$ Der Wunsch nach Kooperation und Kommunikation zwischen unterschiedlichen Einrichtungstypen wurde von den Befragten betont.

Ein Grund dafür, warum das Museum für Naturkunde Berlin bereits über $40 \%$ des wissenschaftlichen Outputs des Museums in Open Access publiziert, ist laut Elisa Herrmann und Stefanie Paß nicht zuletzt der Koordinierungsstelle für wissenschaftliches Publizieren zu verdanken. ${ }^{45}$ Diese Stelle ist zuständig für Publikationsverwaltung, Open Access und die Erstinformation zu rechtlichen Aspekten des Publizierens. Durch die kollaborative Erarbeitung der Open-Access-Leitlinie (2019) des Museums sei die

42 Kändler, Ulrike und Elena Di Rosa. „Alternative Finanzierungsmodelle für Open Access - Herausforderung und Chance für Bibliotheken.“ Gehalten auf: Open-Access-Tage 2020 (OAT2020), 16. September 2020, Bielefeld. https://www.conftool.org/openaccesstage2020/ index.php? page=browseSessions\&presentations $=$ show\&search $=\mathrm{K} \%$ C3\%A4ndler (10.11.2020).

43 Shearer u. a. 2020.

44 Roeder, Corinna und Wolf Christoph Seifert. „Regionalbibliotheken als Akteure der Open-Access-Transformation - Ergebnisse einer Umfrage.“ Gehalten auf: Open-Access-Tage 2020 (OAT2020), 16. September 2020, Bielefeld. doi:10.5281/zenodo.4046847.

$45 \mathrm{~Pa}$, Stefanie und Elisa Herrmann. „Open Access als Herausforderung für Museumsbibliotheken.“ Gehalten auf: Open-Access-Tage 2020 (OAT2020), 24. September 2020, Bielefeld. doi:10.5281/zenodo. 4048174. 
Einrichtung einer Koordinierungsstelle die konsequente institutionelle Verstetigung dieses Prozesses gewesen. ${ }^{46}$

Das Projekt „Implementierung von Open Access an den Hochschulen für angewandte Wissenschaften (HAWen) und den Pädagogischen Hochschulen (PHen) in Baden-Württemberg" soll eine nachhaltige Open-AccessKultur in Baden-Württemberg implementieren. ${ }^{47}$ Auch dieses Projekt betonte die Vernetzung zwischen regionalen und nationalen Open-Access-Aktivitäten. Einigkeit zeigt sich in der Annahme, dass die Verbindung von Praxis und Forschung für Fachhochschulen und Pädagogische Hochschulen eine besondere Herausforderung darstellt, die ohne zusätzliche Ressourcen nicht bearbeitet werden kann. Die Praxisorientierung der Fachhochschulen führt außerdem dazu, dass mit externen Firmen zusammengearbeitet wird, die sich oftmals gegen Open Access aussprechen. ${ }^{48}$ In der Schweiz führt das Projekt „OA-EASI - Open Access for Educational and Applied Sciences in Switzerland" eine Bestandsaufnahme der Publikationen an den Schweizer Fachhochschulen und an den Pädagogischen Hochschulen durch, um die Publikationskultur zu analysieren. Erste Ergebnisse zeigen, dass praxisorientierte Fachzeitschriften eine bedeutende Rolle einnehmen und ein sehr hoher Informations- und Beratungsbedarf hinsichtlich Open Access vorhanden ist. ${ }^{49}$ Desiree Maria Stalder und Dirk Verdicchio betonten zudem, dass die Auflagen der Nationalen Open-Access-Strategie für die Schweiz eine Herausforderung für die Fachhochschulen darstellen, denn der Betrieb von Open-Access-Infrastrukturen ist für Fachhochschulen mit weniger Publikationen deutlich teurer als für Universitäten. Deshalb sei eine „partnerschaftliche Lösung“ zwischen Universitäten und Fachhochschulen besonders wichtig, um die Transformation für diverse Bibliotheks- und Hochschultypen zu unterstützen..$^{50}$

46 Paß/Hermann 2020.

47 Reimer, Nadine, Verena Halbherr, Sebastian Ackermann u. a. „Ein Projekt zur Implementierung von Open Access an den Hochschulen für angewandte Wissenschaften (HAW) und den Pädagogischen Hochschulen (PH) in Baden-Württemberg." Gehalten auf: Open-Access-Tage 2020, 16. September 2020. doi:10.5281/zenodo.4035971.

48 Stalder, Desiree Maria und Dirk Verdicchio. „Von BORIS zu ARBOR. Zusammenarbeit von zwei Hochschulen bei Open Access.“ Gehalten auf: Open-Access-Tage 2020 (OAT2020), 16. September 2020, Bielefeld. https://www.conftool.org/openaccesstage2020/in dex.php?page=browseSessions\&form_session=75\#paperID156 (10.11.2020).

49 Rosenkranz, Simone, Marius Stricker, Valérie Andres u. a. „OAEASI - Open Access for Educational and Applied Sciences in Switzerland.“ Gehalten auf: Open Access Tage 2020, 16. September 2020. doi:10.5281/zenodo.4032863.

50 Stalder/Verdicchio 2020.

\section{Forschungsförderung durch die Deutsche Forschungs- gemeinschaft}

Wie auch zuvor bei den Konferenzen in Präsenz, zeigte sich mit über 300 Teilnehmenden in diesem Jahr ein besonders großes Interesse an der Session der Deutschen Forschungsgemeinschaft (DFG). Angela Holzer und Astrid Sänger stellten die komplementären Förderprogramme ab 2021 vor und Juliane Kant schloss mit einem Beitrag über Preprints im wissenschaftlichen Publizieren die Session ab. Das Förderprogramm „Open-Access-Publikationskosten" sieht Mittel für wissenschaftliche Einrichtungen vor, um Artikelgebühren sowie Open-Access-Bücher aller Disziplinen gleichermaßen zu fördern. Langfristiges Ziel ist es, dass die Finanzierung der Open-Access-Artikel, die in DFG-geförderten Projekten entstehen, nach dem „Verursacher-Prinzip“ auch durch die DFG übernommen werden. Gleichzeitig soll durch dieses Programm Transparenz und Strukturbildung gefördert werden. Das Programm erstreckt sich über zwei Förderphasen bis 2027. Die Berechnung des Bedarfs in der ersten Phase basiert auf der Gesamtzahl der Open-Access-Publikationen einer Einrichtung, in der zweiten Phase dann nur noch für Veröffentlichungen, die im Rahmen DFG-geförderter Projekte entstanden sind. Open-Access-Bücher sind allerdings in beiden Phasen nur dann förderfähig, wenn sie im Rahmen DFG-geförderter Forschung entstanden sind. Für Artikelgebühren gibt es keine Förderhöchstgrenze mehr, sondern eine Pauschale wird ausgegeben, die ggf. eine Ko-Finanzierung ermöglicht. Die Mittel können frei genutzt werden, das heißt beispielsweise auch für Konsortien. Für Artikel selbst gibt es allerdings einige Einschränkungen, so darf es sich nicht um Hybrid-Artikel handeln, außer die Zeitschrift ist Bestandteil eines Transformationsvertrags. Das Merkblatt für dieses Programm ist nach den Open-Access-Tagen veröffentlicht worden. ${ }^{51}$ Das Schwester-Programm „Infrastrukturen für wissenschaftliches Publizieren“ besteht aus drei Schwerpunkten für den Auf- und Ausbau von Publikationsinfrastrukturen und adäquaten strukturellen Rahmenbedingungen und wird damit strategisch von der Finanzierung von Open Access entkoppelt. Die Schwerpunkte wurden bereits in den Vorgängerprogrammen adressiert: die Modellentwicklung und Standardisierung von Verträgen und Finanzflüssen, der Auf- und Ausbau offener Publikationsplattformen sowie Techniken und Verfahren für das wissenschaftliche Publizieren.

51 https://www.dfg.de/foerderung/programme/infrastruktur/lis/ lis_foerderangebote/open_access_publikationskosten/ (26.11.2020). 
Juliane Kant stellte schließlich die Ergebnisse einer Studie von Knowledge Exchange zum Thema Preprints vor..$^{52}$ Die Studie basiert auf einer Literaturrecherche und auf Interviews mit Expertinnen und Experten. Zu Beginn des Beitrags erfragte sie unter den Teilnehmenden die Definition von Preprints mit dem Tool Menti. Die Ergebnisse zeigten, dass es durchaus Unschärfen in der Begriffsdefinition bzw. unterschiedliche Perspektiven darauf gibt. Die Studie hat - obgleich sie bereits 2018/19 durchgeführt wurde - mit Blick auf die wissenschaftliche Kommunikation während der COVID-19-Pandemie eine hohe Relevanz. Preprints spielen hier eine zentrale Rolle als Möglichkeit der frühen und raschen Verbreitung von Forschungsergebnissen, die durch Peers kommentiert werden und das ggf. noch vor der Einreichung bei einer renommierten Zeitschrift.

\section{Offener, nachhaltiger und gerechter - was hat das Jahr 2020 gebracht?}

Die Open-Access-Tage 2020 haben erneut deutlich gemacht, wie viel Arbeit noch vor uns liegt. Die für das Jahr 2020 gesetzten Ziele sind zum großen Teil nicht erreicht, Alternativen zu kommerziellen Finanzierungssystemen sind in der deutschsprachigen Open-Access-Community unterentwickelt, und die Diversität an Publikationskulturen, Finanzierungsansätzen und Einrichtungstypen bringt Herausforderungen mit sich, die viel stärker auch auf strategisch-politischer Ebene adressiert werden müssen und die nur durch zusätzliche Ressourcen bearbeitet werden können. Das Ziel eines offeneren, nachhaltigeren und gerechteren Publikationssystems wird wiederum immer dringender. Nicht zuletzt zeigen uns die COVID-19-Pandemie und die Klimakrise, welche Bedeutung der offene Zugang zu Wissen und Forschung auf überregionaler Ebene spielt.

Die virtuelle Version der Open-Access-Tage hat dank des großartigen Engagements der Organisatorinnen und Organisatoren gezeigt, dass die Open-Access-Community bestens auf virtuellen Austausch eingestellt ist. Dennoch fehlte der wahrscheinlich wichtigste Aspekt eines solchen jährlichen Highlights: das soziale Miteinander, die Pausengespräche, das kurze zufällige Reinstolpern in Sessions, die man wegen anderer Programmpunkte nicht

52 Chiarelli, Andrea, Rob Johnson, Emma Richens u.a. „Accelerating scholarly communication. The transformative role of preprints.“ Knowledge Exchange Report (2019). doi:10.5281/zenodo.3357727. besuchen wollte, wo man dann aber doch an der offenen Tür hängen bleibt, der abendliche Schmaus mit guten Getränken, das Kennenlernen neuer Leute, einfach weil man gemeinsam an einem Tisch steht. So stellen wir fest, dass es in den Jahren „echter“ Open-Access-Tage deutlich einfacher war, sich an persönliche Highlights und Erkenntnisse zu erinnern. Interessanterweise verschwimmen im immer gleichen Zoom-Ambiente die einzelnen Beiträge teilweise sehr stark. Die meisten Präsentationen sind auf dem Repository Zenodo abrufbar ${ }^{53}$ und einzelne Aufnahmen von Vorträgen sind über das TIB AV-Portal verfügbar. ${ }^{54}$ Auch an dieser Stelle gilt unser Dank den Organisatorinnen und Organisatoren sowie allen weiteren Unterstützerinnen und Unterstützern, die hier den Zugang und die Nachhaltigkeit unseres virtuellen Diskurses ermöglichen, auf den wir in einigen Jahren zurückblicken können. Die nächsten Open-Access-Tage 2021 finden vom 27. bis 29. September 2021 in Bern statt. Einrichtungen können sich noch bis zum 1. März 2021 für die Ausrichtung der Open-AccessTage 2022 bewerben..$^{55}$

\section{Autoreninformationen}

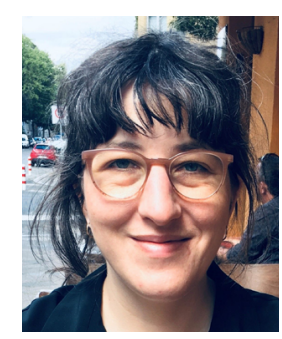

\section{Maike Neufend}

Open-Access-Büro Berlin

c/o Universitätsbibliothek der Freien

Universität Berlin

Ehrenbergstraße 26-28

14195 Berlin

maike.neufend@open-access-berlin.de

orcid.org/0000-0002-1484-0516

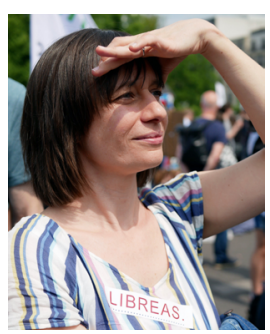

\section{Maxi Kindling}

Open-Access-Büro Berlin

c/o Universitätsbibliothek der Freien

Universität Berlin

Ehrenbergstraße 26-28

14195 Berlin

maxi.kindling@open-access-berlin.de

orcid.org/0000-0002-0167-0466

53 Die Präsentationen und Poster der Open-Access-Tage 2020 sind hier abrufbar: https://zenodo.org/communities/oat2020/?page=1\& size $=20$ (10.11.2020).

54 Die Aufnahmen von Vorträgen sind hier verfügbar: https://av.tib. $\mathrm{eu} /$ search? $\mathrm{q}=$ Open-Access-Tage $+2020 \& \mathrm{f}=$ subject $\% 3 \mathrm{Bhttp} \% 3 \mathrm{~A} \%$ 2F\%2Fav.tib.eu\%2Fresource\%2Fsubject\%2FInformation_Science (10.11.2020).

55 Mehr Informationen dazu finden sich hier: https://open-access. net/?id=773 (8.12.2020). 